\title{
MARKOV CHAIN MODEL OF PHYTOPLANKTON DYNAMICS
}

\author{
RADOSŁAW WIECZOREK \\ Department of Biomathematics \\ Institute of Mathematics, Polish Academy of Sciences ul. Bankowa 14, 40-007 Katowice, Poland \\ e-mail: r.wieczorek@impan.gov.pl
}

\begin{abstract}
A discrete-time stochastic spatial model of plankton dynamics is given. We focus on aggregative behaviour of plankton cells. Our aim is to show the convergence of a microscopic, stochastic model to a macroscopic one, given by an evolution equation. Some numerical simulations are also presented.
\end{abstract}

Keywords: phytoplankton dynamics, coagulation, fragmentation, Markov chain.

\section{Introduction}

Phytoplankton, as the first level of food accessible to animals, is the main source of nutrient in the ocean. That is why the understanding of its behaviour becomes so important. Properties of phytoplankton have been widely investigated by researchers from various branches of science. Phytoplankton cells have the ability to form aggregatesgroups of cells bonded together. Plankton cells in such an aggregate, are joined with a kind of organic glue, called TEP (Passow and Alldredge, 1995). The aggregates undergo diffusion, currents and turbulence, which lead to a dispersion and patchy distribution of phytoplankton in the water.

Since numerical and mathematical modelling plays a crucial role in the understanding of plankton dynamics, there have recently appeared different approaches to the description of plankton with the use of various mathematical methods. One of the approaches uses advectiondiffusion-reaction equations, which describe the spatial densities of cells concentrations (Franks, 2002; Levin and Segel, 1976). In some models the process of the coagulation of plankton cells is included (Laurençot and Mischler, 2002), and these are of great interest for us. An extensive survey of mathematical models of coagulation is given by Aldous (1999). Another approach is based on individual behaviour of cells. It assumes that single cells undergo some random movement and they somehow interact with others. This may lead to the so-called superprocesses (Adler, 1997; Young et al., 2001)), but in our case such a model is related to a description by means of fragmentation-coagulation equations.
We consider here an individual-based model, where a plankton aggregate plays the role of an individual unit. It is a discrete-time, simulation-oriented version of a model presented by Rudnicki and Wieczorek (2006b). It describes a population of aggregates of plankton cells structured by mass and location in the water. The movement of the aggregates is described by a random walk. The proliferation of cells in the aggregates results in the growth of the latter. The fragmentation of the aggregates, as well as their coagulation (i.e., joining together), is included. The model is introduced in Section 2 (and Appendix A). The idea of structuring the plankton population according to the size of aggregates comes here from Arino and Rudnicki (2004), but it was used before, e.g., by Jackson (1990). We present also a macroscopic model in which the mass-spatial distribution of plankton aggregates is described by an evolution equation of the fragmentationcoagulation type. The connection between the micro- and macroscopic models is stressed, namely, we show (in Section 3 with Appendices $B$ and $C$ the limit passage from the first model to the second one as the number of aggregates tends to infinity. An extensive survey of phytoplankton models may be found in the work of Rudnicki and Wieczorek (2008).

Rudnicki and Wieczorek (2006b) presented some results of simulations of a simplified version of their model without coagulation. A numerical individual-based model of phytoplankton with simulations was also presented by El Saadi and Bah (2007). However, no possibility of the aggregation of plankton cells was built into this model: the cells drift in the water totally independently. In this paper 
we conduct some simulations of the discrete-time model, precisely defined in Section 2, in two scenarios, namely, with and without coagulation. Our aim is to observe the behaviour of discrete-time models and to investigate the influence of the coagulation. The results of the simulations are given in Section 5 .

\section{Individual model of plankton dynamics}

Rudnicki and Wieczorek (2006a) constructed a stochastic individual-based model of phytoplankton dynamics, which is the base for the present model. In that model an individual is an aggregate consisting of some number of cells. Each aggregate is located at some point of the three dimensional space $\mathbb{R}^{3}$ and its mass is described by the positive number $m \in \mathbb{R}_{+}$. The proliferation of cells in an aggregate and their mortality result in the growth or decrease of the aggregate. Moreover, the movement of aggregates is modelled by a diffusion process. An aggregate may shatter into two smaller aggregates or may vanish, because of death, sinking or grazing. The aggregates undergo also coagulation, namely, each two of them may join up creating a bigger one. The whole process is described as follows:

(a) The growth rate is $\lambda(m)$. This means that an aggregate grows according to the equation

$$
m^{\prime}=\lambda(m)
$$

(b) An aggregate with mass $m$ moves according to the Brownian motion $2 D(m) W(t)$, where $W(t)$ is a standard Wiener process in $\mathbb{R}^{3}$ and $D(m)$ is the diffusion rate depending on the aggregate size.

(c) An aggregate may die at some time and the death rate is $\lambda_{d}(m)$.

(d) A fraction $\lambda_{f}(m)$ of aggregates of mass $m$ undertake a breakup in a unit of time.

(e) Two aggregates may join together and the probability rate $\kappa$ of coagulation depends on the mass and the locations of both aggregates, and on the state of the whole population.

In this paper we consider a discrete-time version of the above model, which may be directly implemented as a numerical scheme. To this end, we replace the continuous space of positions $\mathbb{R}^{3}$ and masses $\mathbb{R}_{+}$by the lattices, respectively, $\Delta x \cdot \mathbb{Z}^{3}$ and $\Delta m \cdot \mathbb{N}$. This means that $\Delta x$ is the size of the step of movement, whereas $\Delta m$ may be regarded as the mass of a single cell in an aggregate. To formalise mathematically the model, we construct a Markov chain $\xi(n)$ such that in each time step one of the events mentioned above may happen, namely, growth, movement, death, fragmentation or coagulation of some aggregate.

The phase space of the process is defined in a similar manner as in the works of Rudnicki and Wieczorek (2006b) as well as Wieczorek (2007), namely, it is the space $\mathcal{N}$ of counting measures on $\mathbb{R}^{3} \times \mathbb{R}_{+}$of the form

$$
\mathcal{N}=\left\{\nu=\sum_{i=1}^{k_{\nu}} \delta_{x_{i}, m_{i}}: k_{\nu} \in \mathbb{N},\left(x_{i}, m_{i}\right) \in \mathbb{R}^{3} \times \mathbb{R}_{+}\right\}
$$

Consequently, the process state of is described by a finite number of Dirac deltas $\delta_{x_{i}, m_{i}}$, each of which represents a plankton aggregate of mass $m_{i}$ located at $x_{i}$. The whole measure $\nu$ is the so-called empirical distribution of the population of aggregates. Remember that in our model, $m_{i}=n \Delta m$ and $x_{i}=\mathbf{y} \Delta x$ for some $n \in \mathbb{N}$ and $\mathbf{y} \in \mathbb{Z}^{3}$. The process starts from a given state $\nu_{0} \in \mathcal{N}$ consisting of $k_{\nu_{0}}$ aggregates and works as follows: after each time step (which lasts $\Delta t$ ),

(a) an aggregate may grow by $\Delta m$ with probability $\lambda_{p}(n \cdot \Delta m) n$, where $n$ is the number of cells in the aggregate (proliferation of cells) or it may decrease with probability $\lambda_{m}(n \cdot \Delta m) n$ (mortality of cells). Note that the probability coefficients depend on the mass of the aggregate;

(b) an aggregate may jump to one of the neighbouring sites of the lattice with probability $\frac{1}{\Delta x^{2}} D(n \Delta m)$ (random walk, which models the diffusive movement);

(c) an aggregate may die with probability $\lambda_{d}(n \Delta m)$;

(d) an aggregate may split up into two smaller ones with probability $\lambda_{f}(n \Delta m)$. The distribution of masses of the descendent aggregates is given by a function $\Delta m q_{f}^{(\Delta m)}(\bar{n} \Delta m, n \Delta m)$, which describes the conditional probability that the splitting aggregate of mass $n \Delta m$ produces offspring of mass $\bar{n} \Delta m$ and $(n-\bar{n}) \Delta m$. The convention that $q_{f}^{(\Delta m)}$ is multiplied by $\Delta m$ is important in the rescaling forthcoming in the next section;

(e) two aggregates may join together (coagulate) with probability $\lambda_{c}$ depending on the positions and masses of both of them and on the state of the whole population. We claim (cf. Arino and Rudnicki, 2004; Rudnicki and Wieczorek, 2006a; Wieczorek, 2007) that the coagulation rate of an aggregate is $c(n \Delta m)$, and then the aggregate "chooses" another aggregate to coagulate with, according to the coagulation rate of the second one and the distance between them. Therefore, we set

$$
\lambda_{c}=c\left(m_{1}\right) v\left(x_{1}-x_{2}\right) \frac{c\left(m_{2}\right)}{\sum_{i} c\left(m_{i}\right)},
$$


where the sum extends over all living aggregates. We assume that the new aggregate appears in the middle between the two previous ones. One can consider a more general model of the coagulation (cf. Rudnicki and Wieczorek, 2006b);

(f) if none of the above happens, the state of the system remains unchanged.

Obviously, the total probability of the events (a)-(f) for all existing aggregates has to be equal to one. That is why the probability that nothing happens is

$$
\begin{aligned}
1-\sum_{i=1}^{k_{\nu}}[ & \left(\lambda_{p}\left(n_{i} \Delta m\right)+\lambda_{m}\left(n_{i} \Delta m\right)\right) n_{i}+\frac{1}{\Delta x^{2}} D\left(n_{i} \Delta m\right) \\
& \left.+\lambda_{d}\left(n_{i} \Delta m\right)+\lambda_{f}\left(n_{i} \Delta m\right)\right]-\sum_{\substack{i, j=1 \\
i \neq j}}^{k_{\nu}} \lambda_{c}, \quad \text { (4) }
\end{aligned}
$$

which should not be negative. Therefore, the subtracted term has to be bounded by one. If we think about a model that can be fed into a computer, it is natural to assume that we have some maximal number of aggregates, say $K$, and a maximal number of cells in an aggregate, say $M$. In such a setting, we assume that

$$
\begin{aligned}
\bar{\kappa}=K\left[\left(\left\|\lambda_{p}\right\|+\right.\right. & \left.\left\|\lambda_{m}\right\|\right) M+\left\|\lambda_{d}\right\| \\
& \left.+\|D\|+\left\|\lambda_{f}\right\|+K\left\|\lambda_{c}\right\|\right] \leq 1
\end{aligned}
$$

Clearly, $\bar{\kappa}$ is greater than (4). A mathematically precise definition of this Markov chain is given in Appendix A

\section{Passage from micro to macro}

In an individual-based model, a stochastic process describes each individual, even though we have millions of them (in fact, we can observe about 10 million of plankton cells per liter during a plankton bloom). It is hardly possible to study such a model with such a huge number of particles either analytically or numerically by means of computer simulation. Nevertheless, it seems natural to investigate its behaviour in the limit of infinitely many cells.

Therefore, we consider a sequence $\left\{\xi_{N}(n)\right\}_{N \in \mathbb{N}}$ of processes as specified in the previous section, such that the number of aggregates at the beginning tends to infinity. At the same time we go to the "continuity limit", i.e., we assume that the time step $\Delta t$ tends to zero and that the mass-space lattice spacing, namely, $\Delta m$ and $\Delta x$, tend to zero. The transition functions $\kappa_{N}$ of the processes $\xi_{N}$ are given in Appendix B We assume that the rescaled initial distributions $1 / N \xi_{N}(0)$ of plankton aggregates tend to some finite measure $\xi_{\infty}(0)$ on the space $\mathbb{R}^{3} \times \mathbb{R}_{+}$. We have the following result.
Theorem 1. The sequence of processes $t \mapsto \frac{1}{N} \xi_{N}(\lfloor N t\rfloor)$ converges in distribution to a (non-random) measurevalued function $\xi_{\infty}$, i.e., $\xi^{\infty}:[0, \infty) \rightarrow \mathcal{M}\left(\mathbb{R}^{3} \times \mathbb{R}_{+}\right)$, determined by the condition (26), the same as obtained in Theorem 1 of Rudnicki and Wieczorek (2006a).

$\mathcal{M}\left(\mathbb{R}^{3} \times \mathbb{R}_{+}\right)$denotes the space of all finite Borel measures on $\mathbb{R}^{3} \times \mathbb{R}_{+}$. The proof of the theorem is given in Appendix C The measures $\xi_{\infty}(t)$ describe, in a sense, the average dynamics of the population of plankton aggregates. If the initial distribution $\xi_{\infty}(0)$ is absolutely continuous with respect to the Lebesgue measure on $\mathbb{R}^{3} \times \mathbb{R}_{+}$, then the measures $\xi_{\infty}(t)$ are also absolutely continuous for all $t>0$. In such a case one can derive the equation on the densities of $\xi_{\infty}$, which is given in the next section. The process $\xi_{\infty}$ which is the limit in Theorem 1 is identical to that obtained by Rudnicki and Wieczorek (2006a), and the derivation of the equation on densities is given there. Hence we do not rewrite the reasoning here.

\section{Evolution equation for distributions}

In the macroscopic approach, densities of distribution of plankton aggregates in the space of positions and mass are described by means of the following integro-differential equation:

$$
\begin{aligned}
\frac{\partial u(t, x, m)}{\partial t}= & L u(t, x, m)+B u(t, x, m) \\
& +F u(t, x, m)+C u(t, x, m)
\end{aligned}
$$

which the operators $L, B, F$, and $C$ given by

$$
\begin{aligned}
& L f(x, m)=D(m) \Delta_{x} f(x, m), \\
& B f(x, m)=-\frac{\partial}{\partial m}[\lambda(m) f(x, m)], \\
& F f(x, m)=\int_{m}^{\infty} \lambda_{f}(\widetilde{m}) f(x, \widetilde{m}) q_{f}(\widetilde{m}, m) \mathrm{d} \widetilde{m} \\
& \quad-\lambda_{f}(m) f(x, m)-\lambda_{d}(m) f(x, m), \\
& C f(x, m) \quad \frac{1}{V(f)}\left[\int_{\mathbb{R}^{d}} \int_{\mathbb{R}^{d}} \int_{0}^{m} 2^{d} c(m-\bar{m}) c(\bar{m}) v(2(x-\bar{x}))\right. \\
& \quad \times f(\bar{x}, \bar{m}) f(2 x-\bar{x}, m-\bar{m}) \mathrm{d} \bar{x} \mathrm{~d} \bar{m} \\
& \left.-\int_{\mathbb{R}^{d}} \int_{0}^{\infty} 2 c(m) c(\bar{m}) v(x-\bar{x}) f(x, m) f(\bar{x}, \bar{m}) \mathrm{d} \bar{m} \mathrm{~d} \bar{x}\right],
\end{aligned}
$$

where $V(f)=\int_{\mathbb{R}^{d}} \int_{0}^{\infty} c(m) f(x, m) \mathrm{d} x \mathrm{~d} m$ and $\Delta_{x}$ is the Laplace operator with respect to the spatial variable $x$. Equation (6) is obtained as the limit description of the individual-based processes.

We write $u(t)(x, m)$ instead of $u(t, x, m)$, and we consider solutions $u(t)$ of (6) as functions on $\mathbb{R}^{+}$with values in the set $X_{+}$, where $X_{+}$is the subset of all non-negative and non-zero functions from the space 
$X=L^{1}\left(\mathbb{R}^{d} \times \mathbb{R}^{+}\right)$. Equation (6) can be treated as an evolution one

$$
u^{\prime}(t)=(L+B+F+C) u(t) .
$$

Theorem 2. (Rudnicki and Wieczorek, 2006a, Theorem 2) Let all coefficients in the definitions (7)-(10) be sufficiently smooth and let $D(m)>0$ and $c(m)>0$ for $m \geq 0$. For any $u_{0} \in X_{+}$, there exists a unique solution $u(t)$ of 111 such that $u(0)=u_{0}$.

\section{Results of numerical simulations}

Now we present some numerical results concerning the discrete model. The first attempts at simulation had shown that results depend heavily on the choice of the shape of coefficient functions. We propose here some arbitrary, very simple coefficients, which, however, look pretty natural. We conduct the numerical simulations in two different scenarios, namely, with and without coagulation. The simulations are two-dimensional in space: the space of positions for our simulations is the square lattice of size $10000 \times 10000$ with periodic boundary conditions. The simulations start with 3000 aggregates at random (uniformly distributed) locations on the lattice and of random mass. We set constant growth, fragmentation and death rates, namely, $\lambda_{p}(m)=1, \lambda_{f}(m)=7$ and $\lambda_{d}(m)=5$, and the diffusion rate decreasing with the square root of mass (commonly used in physics), namely, $D(m)=$ $5000 / \sqrt{m}$. In the first scenario we assume no coagulation and thereby we set $c=0$, whilst in the second one we set $c(m)=3 m$ and $v(y)=\max \left\{0,1-(y / 250)^{5}\right\}$. The form of function $v$ assures that only aggregates located in the distance less than 250 may coagulate.

A picture of the initial state (the same for both scenarios) is presented in Fig. 1 The states of the system after $2 \cdot 10^{6}$ steps of simulation are shown in Figs. 2 and 3). The darker a point, the heavier the aggregate denoted by it, cf. the scale at the left edge of the figure. By looking at those pictures only, one cannot say much about the behaviour of aggregates. It seems at first that after some time, there is a lower number of aggregates than at the beginning, but it is not so (see Fig. 4). Instead, we claim that the aggregates form a clustered pattern, and that is why a smaller area is covered by them in Figs. 2 and 3 than in the initial state (Fig. 1).

To measure this clustering behaviour we use the socalled Clark-Evans index (or the nearest neighbour index), which was introduced by Clark and Evans (1954) and is now commonly used in the analysis of spatial patterns in many areas (Henderson, 2003; Illian et al., 2008). This index compares the observed mean nearest neighbour distance to the expected one for a random distribution of individuals. The Clark-Evans index is given by

$$
C E I=\frac{d_{\mathrm{emp}}}{d_{\mathrm{exp}}}
$$

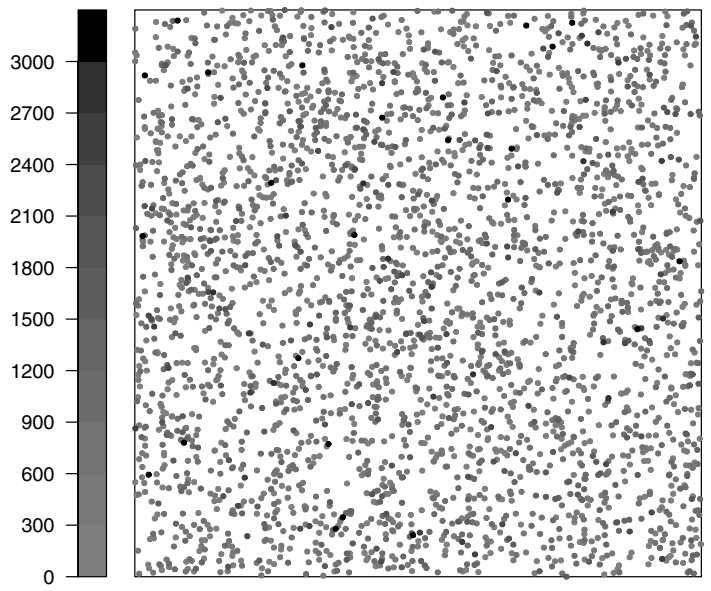

Fig. 1. Initial state of simulation.

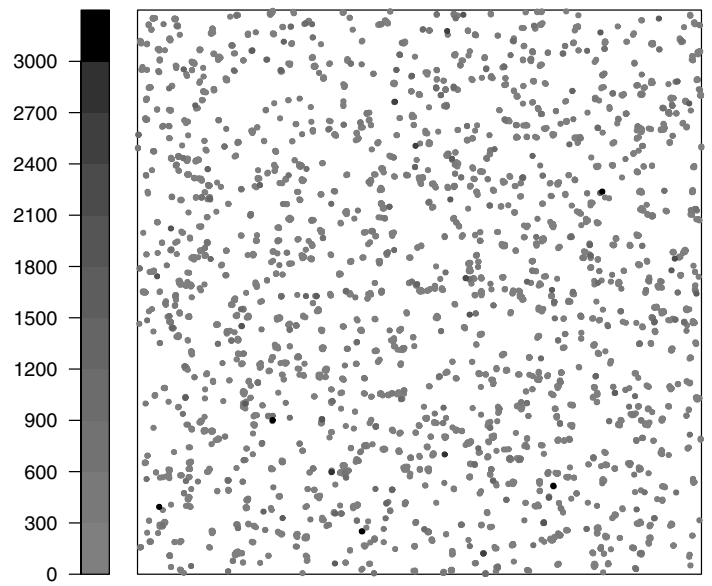

Fig. 2. First scenario: without coagulation, after $2 \cdot 10^{6}$ steps.

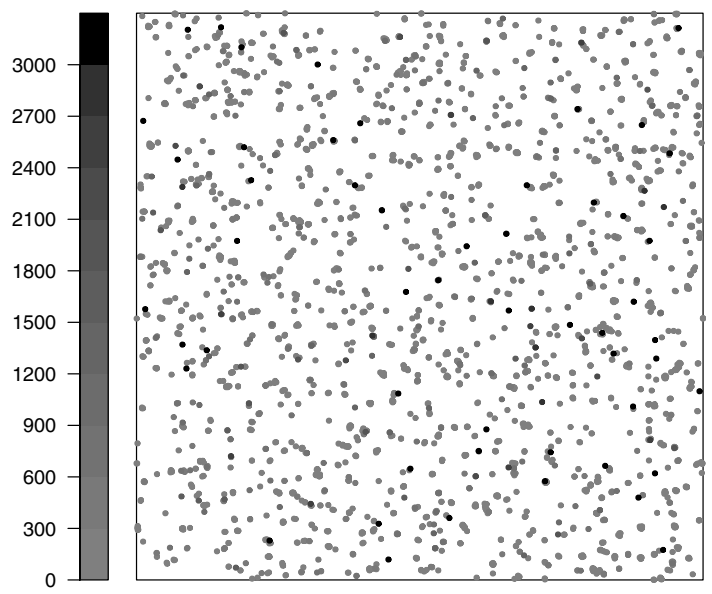

Fig. 3. Second scenario: with coagulation, after $2 \cdot 10^{6}$ steps.

where $d_{\mathrm{emp}}$ and $d_{\mathrm{exp}}$ are, respectively, the empirical and the expected mean distance to the nearest neighbour. If the individuals are located at points $x_{1}, x_{2}, \ldots, x_{N}$, then

$$
d_{\mathrm{emp}}=\frac{1}{N} \sum_{i=1}^{N} \min _{j \neq i}\left(\operatorname{dist}\left(x_{i}, x_{j}\right)\right) .
$$




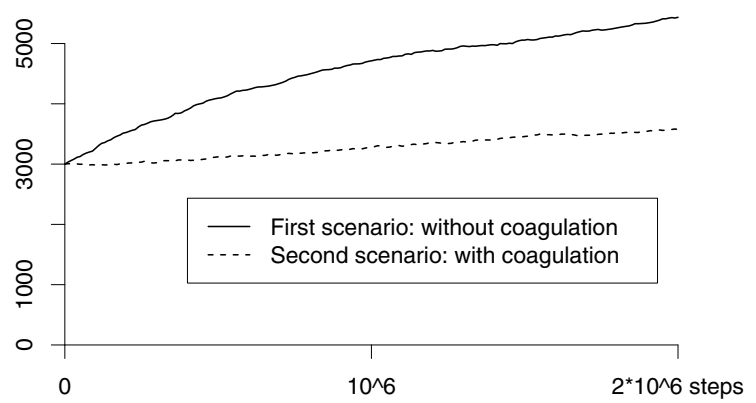

Fig. 4. Total number of aggregates.

The expected mean distance $d_{\exp }$ is the expected value of (13) if the points $x_{1}, x_{2}, \ldots, x_{N}$ are independently and uniformly distributed in some domain $D \subset \mathbb{R}^{d}$. It is assumed that

$$
d_{\exp } \approx \frac{1}{2} \sqrt{\frac{S}{N}},
$$

where $S$ is the area of the domain $D$. It is claimed that the Clark-Evans index indicates a regular, random and clustered pattern of distribution. Namely, if $C E I$ is approximately 1 , then points are randomly distributed. $C E I>1$ means that they have a regular structure and $C E I<1$ suggests that they form a clustered pattern.

Our simulations show that the Clark-Evans index significantly decreases in both scenarios (see Fig. 5], which suggests that, indeed, we eventually obtain a clustered structure.

To compare the cases without and with coagulation, first note that the coagulation clearly leads to a reduction in the number of aggregates, which can be seen in Fig. 4. It is also obvious that larger masses may result from the coagulation, which concerns both the maximal mass of an aggregate (Fig. 7) and the mean mass (Fig. 6). Although really natural, it is quite important, because aggregates rather quickly get very small without coagulation and very fast proliferation of cells is required to avoid this. It is apparent that the coagulation helps to prevent degradation of bigger aggregates. We can see in Fig. 5 that the ClarkEvans index is lower in the case without coagulation. One can ask whether the differnce is statistically signifficant. Although we have not performed any strict statistical test, we have conducted our simulation many times and the results were pretty much the same. Usually, the standard deviation of $d_{\mathrm{emp}}$ is used as a measure of signifficance for the Clark-Evans test (Clark and Evans, 1954). Its value is marked in Fig. 5 by a horizontal bar near the points, and one can see it is very small in proportion to the value of $C E I$. The difference in the value of the Clark-Evans index seems less self-explanatory. Nevertheless, this difference may be explained by the fact that those aggregates which stay close in the scenario without coagulation (and

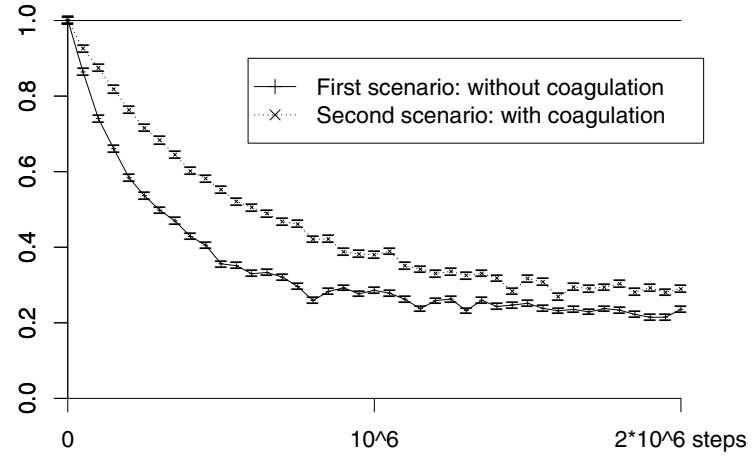

Fig. 5. Clark-Evans index.

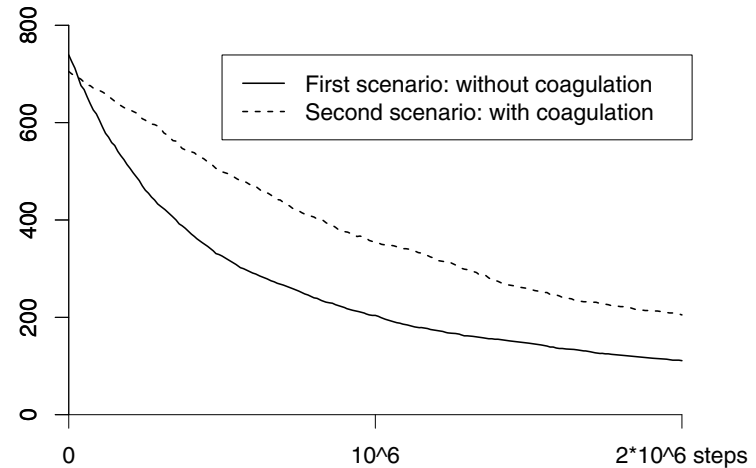

Fig. 6. Mean mass of aggregates.

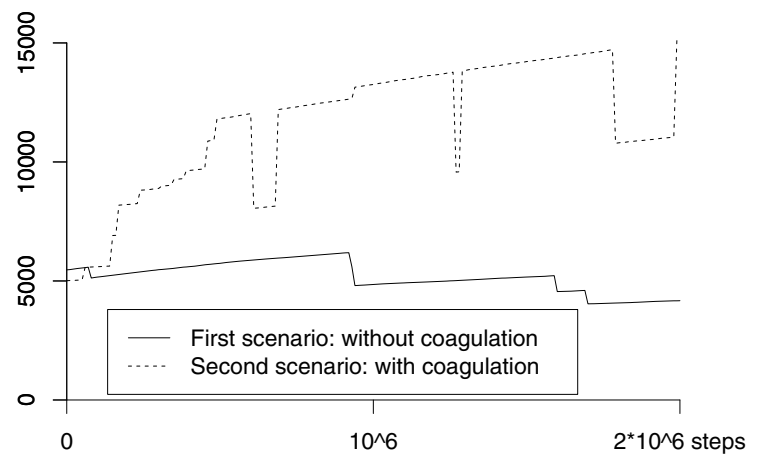

Fig. 7. Mass of the largest aggregate.

result in the decreasing of the nearest-neighbour distance) have a tendency to coagulate in the second scenario, and they join together in one, thereby losing their influence on the Clark-Evans index.

\section{Conclusions}

A discrete-time version of a model of phytoplankton dynamics has been introduced. The model is simula- 
tion oriented, which means that it can be directly implemented as a numerical algorithm. At the same time, for large numbers of plankton cells the model is equivalent to the previous one given by Rudnicki and Wieczorek (2006a; 2006b), which is rigorously confirmed by Theorem 11. It also means that there is a strict connection with the macroscopic model from Section 4 As a result, the macroscopic model is precisely derived from basic interactions between cells and simple rules governing the behaviour of single aggregates. By the way, the model is described by a very interesting mathematical object, studying of which demands the use of involved tools.

In the work of Rudnicki and Wieczorek (2006b), some simulations of a similar model were performed. Nevertheless, they were rather heuristically introduced and did not allow for the coagulation. Here we have a model that enables a direct simulation. The numerical simulations confirmed aggregative behaviour of phytoplankton cells, which is indicated by the Clark-Evans index. The impact of the coagulation on the size structure of the population was also shown: the coagulation ensures the existance of bigger aggregates though the cellproliferation rate is the same.

Plankton aggregates are represented here by point masses. It would be interesting to build a model in which aggregates have finite size and occupy some area or volume. That would require a different coagulation model, which should take into account the possibility of immediate coagulation at the moment the meeting of two aggregates.

\section{Acknowledgment}

The results were presented at the 15 th National Conference on Application of Mathematics to Biology and Medicine held in Szczyrk, Poland, in 2009.

This research was partially supported by the Polish Ministry of Science and Higher Education under Grant No. N N201 021133.

\section{References}

Adler, R. (1997). Superprocesses and plankton dynamics, Monte Carlo Simulation in Oceanography: Proceedings of the 'Aha Huliko'a Hawaiian Winter Workshop, Manoa, HI, pp. 121-128.

Aldous, D. (1999). Deterministic and stochastic models for coalescence (aggregation and coagulation): A review of the mean-field theory for probabilists, Bernoulli 5(1): 3-48.

Arino, O. and Rudnicki, R. (2004). Phytoplankton dynamics, Comptes Rendus Biologies 327(11): 961-969.

Clark, P. and Evans, F. (1954). Distance to nearest neighbor as a measure of spatial relationships in populations, Ecology 35(4): 445-453.
El Saadi, N. and Bah, A. (2007). An individual-based model for studying the aggregation behavior in phytoplankton, Ecological Modelling 204(1-2): 193-212.

Ethier, S.N. and Kurtz, T.G. (1986). Markov Processes: Characterization and Convergence, Wiley Series in Probability and Mathematical Statistics: Probability and Mathematical Statistics, John Wiley \& Sons, Inc., New York, NY.

Franks, P.J.S. (2002). NPZ models of plankton dynamics: Their construction, coupling to physics, and application, Journal of Oceanography 58(2): 379-387.

Henderson, P.A. (2003). Practical Methods in Ecology, WileyBlackwell, Malden, MA.

Illian, J., Penttinen, A., Stoyan, H. and Stoyan, D. (2008). Statistical Analysis and Modelling of Spatial Point Patterns, John Wiley \& Sons Ltd, Chichester.

Jackson, G. (1990). A model of the formation of marine algal flocs by physical coagulation processes, Deep-Sea Research 37(8): 1197-1211.

Laurençot, P. and Mischler, S. (2002). The continuous coagulation-fragmentation equations with diffusion, Archive for Rational Mechanics and Analysis 162(1): 45-99.

Levin, S.A. and Segel, L.A. (1976). Hypothesis for origin of planktonic patchiness, Nature 259.

Passow, U. and Alldredge, A. (1995). Aggregation of a diatom bloom in a mesocosm: The role of transparent exopolymer particles (TEP), Deep-Sea Research II 42(1): 99-109.

Rudnicki, R. and Wieczorek, R. (2006a). Fragmentationcoagulation models of phytoplankton, Bulletin of the Polish Academy of Sciences: Mathematics 54(2): 175-191.

Rudnicki, R. and Wieczorek, R. (2006b). Phytoplankton dynamics: from the behaviour of cells to a transport equation, Mathematical Modelling of Natural Phenomena 1(1): 83-100.

Rudnicki, R. and Wieczorek, R. (2008). Mathematical models of phytoplankton dynamics, Dynamic Biochemistry, Process Biotechnology and Molecular Biology 2 (1): 55-63.

Wieczorek, R. (2007). Fragmentation, coagulation and diffusion processes as limits of individual-based aggregation models, Ph.D. thesis, Institute of Mathematics, Polish Academy of Sciences, Warsaw, (in Polish).

Young, W., Roberts, A. and Stuhne, G. (2001). Reproductive pair correlations and the clustering of organisms, Nature 412(6844): 328-331.

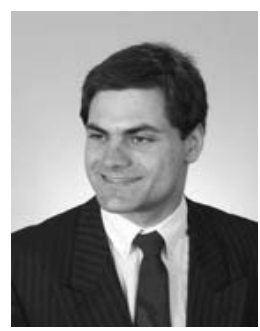

Radosław Wieczorek was born in 1980 in Bytom. He obtained his M.Sc. degree from Silesian University, Katowice, Poland, and his Ph.D. degree from the Institute of Mathematics of the Polish Academy of Sciences. His research interests include stochastic processes, mathematical modelling and applications of mathematics in biology. Currently, he is an assistant professor at the Institute of Mathematics of the Polish Academy of Sciences. 


\section{Appendix A}

Construction of the Markov chain. We assume that the phase space of our process $\xi$ is the space of measures $\mathcal{N}$ defined by the formula (2). The process starts from some given state $\xi(0) \in \mathcal{N}$, which consists of $k_{0}=\xi(0)\left(\mathbb{R}^{3} \times \mathbb{R}_{+}\right)$aggregates (remember that the states of the process are measures and $\xi(t)(A)$ denotes the number of aggregates with positions and masses in the set $A \subset \mathbb{R}^{3} \times \mathbb{R}_{+}$at time $t$ ). To define a Markov chain we need to specify a transition function, i.e., a family of measures that describe probabilities of transitions from one state to another. Let us first define a measure that describes the events: proliferation and mortality of cells, random walk, death, fragmentation and coagulation of aggregates (in that order).

$$
\begin{aligned}
& \kappa_{\text {full }}(\nu, B) \\
& =\sum_{i=1}^{k_{\nu}}\left[\lambda_{p}\left(m_{i}\right) \frac{m_{i}}{\Delta m} \mathbb{1}_{B}\left(\nu-\delta_{x_{i}, m_{i}}+\delta_{x_{i}, m_{i}+\Delta m}\right)\right. \\
& \quad+\lambda_{m}\left(m_{i}\right) \frac{m_{i}}{\Delta m} \mathbb{1}_{B}\left(\nu-\delta_{x_{i}, m_{i}}+\delta_{x_{i}, m_{i}-\Delta m}\right) \\
& \quad+\frac{1}{\Delta x^{2}} D\left(m_{i}\right) \sum_{l=1}^{6} \mathbb{1}_{B}\left(\nu-\delta_{x_{i}, m_{i}}+\delta_{x+\varepsilon_{i} \Delta x, m_{i}}\right) \\
& \quad+\lambda_{d}\left(m_{i}\right) \mathbb{1}_{B}\left(\nu-\delta_{x_{i}, m_{i}}\right) \\
& \quad+\lambda_{f}\left(m_{i}\right) \sum_{l=0}^{\frac{m_{i}}{\Delta m}} \mathbb{1}_{B}\left(\nu-\delta_{x_{i}, m_{i}}+\delta_{x_{i}, l \Delta m}\right. \\
& \left.\left.\quad+\delta_{x_{i}, m_{i}-l \Delta m}\right) \times \Delta m q_{f}^{(\Delta m)}\left(l \Delta m, m_{i}\right)\right] \\
& \quad+\sum_{\substack{i, j=1 \\
i \neq j}}^{k_{\nu}} \lambda_{c} \mathbb{1}_{B}\left(\nu-\delta_{x_{i}, m_{i}}-\delta_{x_{j}, m_{j}}+\delta_{x, m_{i}+m_{j}}\right),
\end{aligned}
$$

where

$$
\nu=\sum_{i=1}^{k_{\nu}} \delta_{x_{i}, m_{i}},
$$

and $\varepsilon_{i}, i=1, \ldots, 6$ denote the unit vectors parallel to each of the three axes and positively or negatively directed. Note that $\kappa_{\text {full }}$ depends on the parameters $\Delta m$ and $\Delta x$. We assume that all functional coefficients, namely, $\lambda_{p}, \lambda_{m}, D$, $\lambda_{d}, \lambda_{f}$, and $\lambda_{c}$, are smooth and non-negative. Recalling also the formula (3), we assume that $v$ and $c$ are smooth and $c$ is strictly positive. Since $\Delta m q_{f}^{(\Delta m)}(\bar{m}, m)$ is a conditional probability that the splitting aggregate produces offspring of mass $\bar{m}$ (where both $m$ and $\bar{m}$ are multiples of $\Delta m$ ), we shall assume that

$$
\sum_{i=0}^{\frac{m}{\Delta m}} \Delta m q_{f}^{(\Delta m)}(i \Delta m, m)=1 .
$$

Notice also that

$$
\kappa_{\text {full }}(\nu, \mathcal{N}) \leq \bar{\kappa} \leq 1
$$

with $\bar{\kappa}$ defined by (5). Now we are ready to write the transition function of the Markov chain described in Section 2. Namely,

$$
\begin{aligned}
& \kappa(\nu, B) \\
& = \begin{cases}\kappa_{\text {full }}(\nu, B)+\left[1-\kappa_{\text {full }}(\nu, \mathcal{N})\right] \mathbb{1}_{B}(\nu) \\
\text { if } k_{\nu} \leq K \text { and } m_{i} \leq M \text { for } i=1, \ldots, k_{\nu} \\
0, \quad \text { otherwise. }\end{cases}
\end{aligned}
$$

\section{Appendix B}

Sequence of Markov chains. Let us now construct a sequence of Markov chains $\xi_{N}(n)$ based on the one defined above. We assume that the $N$-th chain starts with the state $\xi_{N}(0)$ which consists exactly of $N$ aggregates, so $k_{N, 0}=$ $\xi_{N}(0)(X)=N$, and that these initial empirical distributions approximate some distribution of aggregates in the mass-position space, precisely, $\frac{1}{N} \xi_{N}(0) \underset{N \rightarrow \infty}{\longrightarrow} \xi_{\infty}(0)$, where $\xi_{\infty}(0)$ is some finite Borel measure on $\mathbb{R}^{3} \times \mathbb{R}_{+}$. Moreover, we assume that in the $N$-th process the lattice spacing is $\Delta m=1 / N$ and $\Delta x=1 / N$. We also let $\Delta t$ be $1 / N$ and thereby we rescale the whole transition function by $1 / N$ so that the probability rates in a unit of time are preserved. Moreover, since now the probability of a single event in one step is smaller, we can allow for greater sizes of aggregates $M_{N}$ and a greater maximal number of aggregates $K_{N}$. We, thereby, assume that $M_{N} \rightarrow \infty$ and $K_{N} \rightarrow \infty$ as $N$ tends to $\infty$ in such a way that

$$
\begin{aligned}
\bar{\kappa}_{N}= & \frac{1}{N} K_{N}\left[\left(\left\|\lambda_{p}\right\|+\left\|\lambda_{m}\right\|\right) M_{N}+\left\|\lambda_{d}\right\|+\|D\|\right. \\
& \left.+\left\|\lambda_{f}\right\|+K_{N}\left\|\lambda_{c}\right\|\right]
\end{aligned}
$$

is less than one. Thus the transition functions of the chains in the sequence are given by

$$
\begin{aligned}
& \kappa_{N}(\nu, B) \\
& =\left\{\begin{array}{l}
\frac{1}{N} \kappa_{\text {full }}(\nu, B)+\left[1-\frac{1}{N} \kappa_{\text {full }}(\nu, \mathcal{N})\right] \mathbb{1}_{B}(\nu), \\
\quad \text { if } k_{\nu} \leq K_{N} \text { and } m_{i} \leq M_{N} \text { for } i=1, \ldots, k_{\nu}, \\
0, \quad \text { otherwise }
\end{array}\right.
\end{aligned}
$$

with $\Delta m$ an $\Delta x$ replaced by $\frac{1}{N}$ in $\kappa_{\text {full }}$, cf. the formula (15). We assume also that $q_{f}^{(1 / N)}(\cdot, m)$ converges uniformly to some smooth function $q_{f}(\cdot, m):[0, m] \rightarrow \mathbb{R}$; cf. the discussion on $q_{f}^{(\Delta m)}$ and notice that the condition (17) becomes in the limit

$$
\int_{0}^{m} q_{f}(\bar{m}, m) \mathrm{d} \bar{m}=1 .
$$

\section{Appendix C}

Proof of Theorem 1, Let $\|\cdot\|$ denote the measure of the whole space in the case of measure and the supremum norm for functions, respectively: $\|\nu\|=\nu\left(\mathbb{R}^{3} \times \mathbb{R}_{+}\right)$and $\|f\|=\sup _{x}|f(x)|$. 
Lemma 1. Consider a Markov chain $\left(\xi_{N}(i)\right)_{i \in \mathbb{N}}$ with the transition function $\kappa_{N}$ defined in the previous section. We have

$$
\begin{aligned}
\operatorname{Prob}\left(\max _{0 \leq i / N \leq t}\left\|\frac{1}{N} \xi_{N}(i)\right\|\right. & \geq a) \\
& \leq \frac{1}{a} e^{\left\|\lambda_{f}\right\| t} \mathrm{E}\left\|\frac{1}{N} \xi_{N}(0)\right\| .
\end{aligned}
$$

Proof. First, let us prove that a process

$$
-\left\|\xi_{N}(i)\right\|\left(1+\frac{1}{N}\left\|\lambda_{f}\right\|\right)^{-i}
$$

is a sub-martingale. Indeed, observe that $\left\|\xi_{N}(i)\right\|$ may increase at most by one, in every step. The conditional probability that $\left\|\xi_{N}(i+1)\right\|-\left\|\xi_{N}(i)\right\|=1$ is not greater than $\frac{1}{N}\left\|\xi_{N}(i)\right\|\left\|\lambda_{f}\right\|$. Therefore,

$$
\begin{aligned}
& \mathrm{E}\left(\left\|\xi_{N}(i+1)\right\| \mid \mathcal{F}_{i}\right) \\
& \quad=\left\|\xi_{N}(i)\right\|+\mathrm{E}\left(\left\|\xi_{N}(i+1)\right\|-\left\|\xi_{N}(i)\right\| \mid \mathcal{F}_{i}\right) \\
& \leq\left\|\xi_{N}(i)\right\|+\frac{1}{N}\left\|\xi_{N}(i)\right\|\left\|\lambda_{f}\right\| .
\end{aligned}
$$

Hence,

$$
\begin{aligned}
\mathrm{E}\left(-\left(1+\frac{1}{N}\left\|\lambda_{f}\right\|\right)^{-(i+1)}\right. & \left.\left\|\xi_{N}(i+1)\right\| \mid \mathcal{F}_{i}\right) \\
& \geq-\left(1+\frac{1}{N}\left\|\lambda_{f}\right\|\right)^{-i}\left\|\xi_{N}(i)\right\|,
\end{aligned}
$$

so 23 is a negative sub-martingale.

Second, let us make use of the Doob-Kolmogorov inequality in the version from Ethier and Kurtz (1986, Lemma 2.3, Chapter 2).

$$
\begin{aligned}
& \operatorname{Prob}\left(\max _{0 \leq i \leq n}\left\|\xi_{N}(i)\right\| \geq a\right) \\
& =\operatorname{Prob}\left(\min _{0 \leq i \leq n}-\frac{\left\|\xi_{N}(i)\right\|}{\left(1+\frac{1}{N}\left\|\lambda_{f}\right\|\right)^{n}} \leq-\frac{a}{\left(1+\frac{1}{N}\left\|\lambda_{f}\right\|\right)^{n}}\right) \\
& \leq \operatorname{Prob}\left(\min _{0 \leq i \leq n}-\frac{\left\|\xi_{N}(i)\right\|}{\left(1+\frac{1}{N}\left\|\lambda_{f}\right\|\right)^{i}} \leq-\frac{a}{\left(1+\frac{1}{N}\left\|\lambda_{f}\right\|\right)^{n}}\right) \\
& \leq \frac{\left(1+\frac{1}{N}\left\|\lambda_{f}\right\|\right)^{n}}{a} \mathrm{E}\left\|\xi_{N}(0)\right\| .
\end{aligned}
$$

Thus,

$$
\begin{aligned}
& \operatorname{Prob}\left(\max _{0 \leq i / N \leq t}\left\|\frac{1}{N} \xi_{N}(i)\right\| \geq a\right) \\
& \leq \frac{1}{a}\left(1+\frac{1}{N}\left\|\lambda_{f}\right\|\right)^{N t} \mathrm{E}\left\|\frac{1}{N} \xi_{N}(0)\right\| \\
& \leq \frac{1}{a} e^{\left\|\lambda_{f}\right\| t} \mathrm{E}\left\|\frac{1}{N} \xi_{N}(0)\right\| .
\end{aligned}
$$

Definition 1. We say that a sequence of processes $\left\{X_{N}\right\}_{N \in \mathbb{N}}$ satisfies a compact containment condition if for all $\varepsilon>0$ and $T>0$ there is a compact set $\Gamma_{\varepsilon, T}$ such that

$$
\inf _{N \in \mathbb{N}} \operatorname{Prob}\left\{X_{N}(t) \in \Gamma_{\varepsilon, T}: \quad 0 \leq t \leq T\right\} \geq 1-\varepsilon .
$$

Proposition 1. (Ethier and Kurtz, 1986, Corollary 8.17, Chapter 4) Let $(E, r)$ be a Polish space, $\mathcal{L}: \mathbf{C}_{\mathrm{b}}(E) \supset$ $\mathcal{D}(\mathcal{L}) \rightarrow \mathbf{C}_{\mathrm{b}}(E)$ be an operator, and $P_{0}$ be a probability measure on $E$. Suppose that the martingale problem for $\left(\mathcal{L}, P_{0}\right)$ has at most one solution. For $N=1,2, \ldots$, suppose that $X_{N}(t)=\eta_{N}\left(\xi_{N}\left(\left\lfloor\alpha_{N} t\right\rfloor\right)\right)$ where $\left\{\xi_{N}(k), k=\right.$ $1,2, \ldots\}$ is a Markov chain in a metric space $E_{N}$ with a transition function $\kappa_{N}(x, B)$ and $\eta_{N}: E_{N} \rightarrow E$ is measurable. Let also $\alpha_{N}$ tend to infinity as $N \rightarrow \infty$. Define $T_{N}: \mathbf{B}\left(E_{N}\right) \rightarrow \mathbf{B}\left(E_{N}\right)$ by

$$
T_{N} f(x)=\int_{E_{N}} f(y) \kappa_{N}(x, \mathrm{~d} y),
$$

and let $\mathcal{L}_{N}=\alpha_{N}\left(T_{N}-I\right)$. Suppose, moreover, that the distribution of $X_{N}(0)$ converges weakly to $P_{0}$ as $N \rightarrow$ $\infty$, the sequence $\left\{X_{N}\right\}$ satisfies the compact containment condition (24) and that the closure of the linear span of $\mathcal{D}(\mathcal{L})$ contains an algebra that separates points.

If for all $f \in \mathcal{D}(\mathcal{L})$ and $T>0$ there exists a sequence of functions $f_{N} \in \mathbf{B}\left(E_{N}\right)$ and sets $\Gamma_{N} \subset E_{N}$ such that

(Pla)

$$
\lim _{N \rightarrow \infty} \operatorname{Prob}\left(\left\{\xi_{N}\left(\left\lfloor\alpha_{N} t\right\rfloor\right) \in \Gamma_{N}, 0 \leq t \leq T\right\}\right)=1,
$$

(PIb) $\sup _{N}\left\|f_{N}\right\|<\infty$,

(P1c) $\lim _{N \rightarrow \infty} \sup _{y \in \Gamma_{N}}\left|f \circ \eta_{N}(y)-f_{N}(y)\right|=0$ and

(Pld) $\lim _{N \rightarrow \infty} \sup _{y \in \Gamma_{N}}\left|(\mathcal{L} f) \circ \eta_{N}(y)-\mathcal{L}_{N} f_{N}(y)\right|=0$,

then there exists a solution $X$ of the $\left(\mathcal{L}, P_{0}\right)$ martingale problem and $X_{N}$ converges in distribution to $X$.

Remark 1. The measure-valued function $\xi_{\infty}$, obtained as a limit of discrete models in Theorem 1, is uniquely determined by the equation

$$
\begin{aligned}
& \langle h, \xi(t)\rangle-\left\langle h, \xi_{0}\right\rangle \\
& =\int_{0}^{t}\left[\left\langle\left(L^{*}+B^{*}+F^{*}\right) h, \xi(s)\right\rangle+C^{*}(h, \xi(s))\right] \mathrm{d} s
\end{aligned}
$$

for all $h \in C_{\mathrm{b}}^{2}$, with

$$
\begin{aligned}
L^{*} h(x, m)= & D(m) \Delta_{x} h(x, m), \\
B^{*} h(x, m)= & m\left(\lambda_{p}(m)-\lambda_{m}(m)\right) \frac{\partial}{\partial m} h(x, m), \\
F^{*} h(x, m)= & 2 \lambda_{f}(m) \int_{0}^{m} h(x, \bar{m}) q(m, \bar{m}) \mathrm{d} \bar{m} \\
& -\lambda_{f}(m) h(x, m)-\lambda_{d}(m) h(x, m), \\
C^{*}(h, \xi)= & \iiint \int \frac{c(m) c(\bar{m}) v(x-\bar{x})}{\iint c(\overline{\bar{m}}) \xi(\mathrm{d} \overline{\bar{x}} \mathrm{~d} \overline{\bar{m}})} \\
& \times(h((x+\bar{x}) / 2, m+\bar{m})-h(x, m) \\
& +h(\bar{x}, \bar{m})) \xi(\mathrm{d} x \mathrm{~d} m) \xi(\mathrm{d} \bar{x} \mathrm{~d} \bar{m}) .
\end{aligned}
$$


Proof of Theorem 1 We use Proposition 1, Let us define an operator $\mathcal{L}$ with the formula

$$
\begin{aligned}
& \mathcal{L}[\exp [-\langle h, \nu\rangle]] \\
& =\exp [-\langle h, \nu\rangle] \times\left[\left\langle-L^{*} h-B^{*} h-F^{*} h, \nu\right\rangle\right. \\
& \left.\quad+C^{*}(h, \nu)\right],
\end{aligned}
$$

with $L^{*}, B^{*}, F^{*}$ and $C^{*}$ given by 27 - 30 with the domain

$$
\begin{aligned}
\mathcal{D}(\mathcal{L})=\{ & f_{h} \in \mathbf{C}_{b}(\mathcal{N}): f_{h}(\nu)=\exp [-\langle h, \nu\rangle] \\
& \text { with } h \in \mathcal{D}(L) \cap \mathcal{D}(B) \text { and } h>0\} .
\end{aligned}
$$

We employ here the same trick as found in the works of Rudnicki and Wieczorek (2006a) as well as Wieczorek (2007), namely, we use temporarily the one-point compactification of the space $\mathbb{R}^{3} \times \mathbb{R}_{+}$and denote $\hat{E}=$ $\mathbb{R}^{3} \times \mathbb{R}_{+} \cup\{\infty\}$ with a appropriate metrics. Thus we obtain a limit with values, actually, in $\mathcal{M}\left(\mathbb{R}^{3} \times \mathbb{R}_{+} \cup\{\infty\}\right)$, but all those measures are in fact supported on $\mathbb{R}^{3} \times \mathbb{R}_{+}$ only, which is proved in those papers.

We assign our objects to the notation of Proposition 1 in the following way: Let $E=\mathcal{M}(\hat{E})$ and $E_{N}=\mathcal{N}(\hat{E})$ for all $N \in \mathbb{N}$; moreover, $\eta_{N}(\nu)=\frac{1}{N} \nu$ and $\alpha_{N}=N$, so $X_{N}(t)=\frac{1}{N} \xi_{N}(\lfloor N t\rfloor)$ as we expect.

All assumptions of Theorem 1 concerning the operator $\mathcal{L}$, namely, the uniqueness of a solution to a martingale problem with $(\mathcal{L}, \nu)$ and the condition on the domain, were checked by Rudnicki and Wieczorek (2006a) as well as Wieczorek (2007). The compact containment condition (24) of the sequence $X_{N}$ follows from Lemma1 because all sets of the form $\{\nu \in \mathcal{N}(\hat{E}):\langle 1, \nu\rangle \leq a\}$ are compact. Let us take all the sets $\Gamma_{N}$ in Proposition 1 equal to the whole space $E_{N}=\mathcal{N}(\hat{E})$. Thus the condition (F[1]) is satisfied in an obvious way. Now it suffices to check that for all $f_{h} \in \mathcal{D}(\mathcal{L})$ there exists a sequence $f_{h, N}$ of functions satisfying the conditions (b)-(c) of Proposition 1, To that end, let us define $f_{h, N}(\nu)=\exp \left[\left\langle\log \left(1-\frac{1}{N} h\right), \nu\right\rangle\right]$. Since $h$ is positive, $f_{h, N}$ are bounded by one, which assures ( $[1 b)$. It remains to check (P1c) and (P1d). First, notice that

$$
\begin{aligned}
& \left|f_{h}\left(\frac{1}{N} \nu\right)-f_{h, N}(\nu)\right| \\
& \quad=\left|\exp \left[-\frac{1}{N}\langle h, \nu\rangle\right]-\exp \left[\left\langle\log \left(1-\frac{1}{N} h\right), \nu\right\rangle\right]\right| \\
& \leq\left|\frac{1}{N}\langle h, \nu\rangle+\left\langle\log \left(1-\frac{1}{N} h\right), \nu\right\rangle\right| \\
& \leq \frac{1}{N}\left\langle\left|h+\log \left(1-\frac{1}{N} h\right)^{N}\right|, \nu\right\rangle \exp \left[-\frac{1}{N}\langle h, \nu\rangle\right] \\
& \quad \underset{N \rightarrow \infty}{\longrightarrow} 0
\end{aligned}
$$

uniformly with respect to $\mathcal{N}$. Secondly, one can check that the value of the operator $\mathcal{L}_{N}$, defined as in Proposition 1 , acting on a function $f_{h, N}$ is

$$
\begin{aligned}
\mathcal{L}_{N} f_{h, N} & (\nu) \\
= & \exp \left\langle\log \left(1-\frac{h}{N}\right), \nu\right\rangle \\
& \times\left[\left\langle\frac{L_{N} h+B_{N} h+N F_{N}\left(1-\frac{h}{N}\right)}{1-\frac{h}{N}}, \frac{\nu}{N}\right\rangle\right. \\
& \left.+\mathcal{C}\left(N-h, \frac{\nu}{N}\right)\right],
\end{aligned}
$$

where

$$
\begin{aligned}
& \begin{array}{l}
B_{N} g(x, m) \\
=N m\left[\lambda_{p}(m) g\left(x, m+\frac{1}{N}\right)+\lambda_{m}(m) g\left(x, m-\frac{1}{N}\right)\right] \\
\quad-N m\left(\lambda_{p}(m)+\lambda_{m}(m)\right) g(x, m),
\end{array} \\
& \begin{array}{l}
L_{N} g(x, m)=N^{2} D(m)\left[\sum_{l=1}^{6} g\left(x+\frac{1}{N} \varepsilon_{l}, m\right)-g(x, m)\right], \\
F_{N} g(x, m) \\
\left.=\lambda_{f}(m) \sum_{l=0}^{\frac{m_{i}}{\Delta m}} g\left(x, \frac{l}{N}\right) g\left(x, m-\frac{l}{N}\right)\right) \frac{1}{N} q_{f}^{(1 / N)}\left(l \Delta m, m_{i}\right) \\
\quad-\lambda_{f}(m) g(x, m)+\lambda_{d}(m)[1-g(x, m)],
\end{array}
\end{aligned}
$$

and

$$
\begin{aligned}
& \mathcal{C}(g, \nu) \\
& \quad=\iiint \int \frac{c(m) c(\bar{m})}{\iint c(\overline{\bar{m}}) \nu(\mathrm{d} \overline{\bar{x}} \mathrm{~d} \overline{\bar{m}})} v(x-\bar{x}) \\
& \quad \times\left[\frac{g((x+\bar{x}) / 2, m+\bar{m})}{g(x, m) g(\bar{x}, \bar{m})}-1\right] \nu(\mathrm{d} x \mathrm{~d} m) \nu(\mathrm{d} \bar{x} \mathrm{~d} \bar{m}) .
\end{aligned}
$$

One can observe that $B_{N} \rightarrow B^{*}, L_{N} \rightarrow L^{*}, N F_{N}(1-$ $\left.\frac{h}{N}\right) \rightarrow F^{*}(h)$ and $\mathcal{C}(N-h, \nu) \rightarrow C^{*}(h, \nu)$. A careful calculation shows that $(\mathrm{P} 1 \mathrm{~d})$ is indeed satisfied.

Received: 2 February 2010 Revised: 1 June 2010 